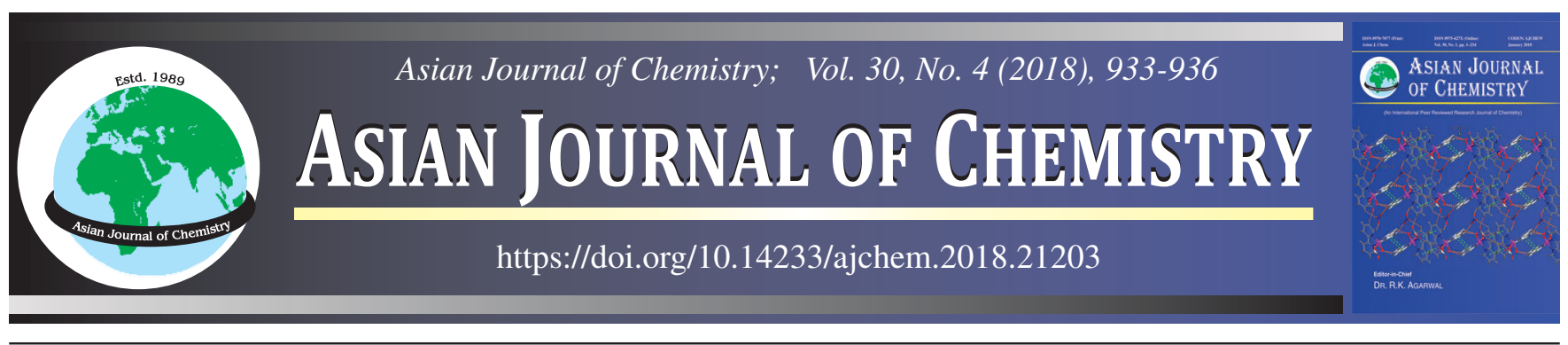

\title{
An ONIOM Study of Catalytically Active Brønsted Acid Sites of ZSM-5
}

\author{
P.S. Arun ${ }^{1,2, *}$, T.K. Manoj Kumar ${ }^{2}$, Revathy B. Nair ${ }^{3}$, S.R. Archana ${ }^{1}$ and S.M.A. Shibli ${ }^{1}$
}

${ }^{1}$ Department of Chemistry, University of Kerala, Kariavattom Campus, Thiruvananthapuram-695 581, India

${ }^{2}$ Indian Institute of Information Technology and Management-Kerala, Technopark Campus, Thiruvananthapuram-695 581, India

${ }^{3}$ Department of Chemistry, Government College, Attingal, Thiruvananthapuram-695 101, India

*Corresponding author: E-mail: arunpsvjmd@gmail.com

Catalytic cracking process is specifically initiated on Brønsted acid site and the loss of strong Brønsted acid site is directly correlated with the loss of catalytic activity. In this context we first identified and established the activity of Brønsted acid sites in three-dimensional ZSM-5 by identifying the preferential occurrence of LUMO orbital at these sites. Critical lowering of HOMO-LUMO energy gap in carbon monoxide adsorbed ZSM-5 compared to free CO, revealed the cause for high activation of CO.

Keywords: ZSM-5 catalyst, Brønsted acid site, ONIOM study, DFT, CO activation, HOMO-LUMO analysis.

ᄂ - - - - - - - - - - - - - - - - -

\section{INTRODUCTION}

Sustainable Brønsted acid properties of ZSM-5 make it catalytically active and important in certain industrial processes including catalytic cracking of hydrocarbons (FCC additive) and conversion of methanol to gasoline [1-3]. Bridged hydroxyl groups [ $\mathrm{Si}-\mathrm{O}(\mathrm{H})-\mathrm{Al}]$ of the ZSM-5 act as strong Brønsted acid sites [2] i.e., the catalytically active centres during those reactions. Accordingly, the catalytic cracking process is specifically initiated on the Brønsted acid site itself while the Brønsted acidity of zeolite particles influences the overall cracking efficiency of the catalyst [3,4]. Buurmans et al. [3] have demonstrated and proved that the loss of strong Brønsted acid site is directly correlated with the loss of catalytic activity during the catalytic cracking process. Their views have been endorsed and applied by extensive researchers. However, the exclusive nature of existence of the sites and their role in loss of catalytic activity has remained as 'yet to be proved' for the past 3 years. We analyzed the role of the LUMO centric site, its activity and loss during the present DFT study.

\section{EXPERIMENTAL}

The three dimensional ZSM-5((3D:ZSM-5) cluster used in this study was modeled as a $\left[\mathrm{AlSi}_{45} \mathrm{H}_{49} \mathrm{O}_{68}\right]$ cluster containing 163 atoms reported by Mynsbrugge et al. [5], the optimized structures are shown in Fig. 1a. The Brønsted acid containing cluster (BASCC) was modeled as $\left[\mathrm{AlSi}_{4} \mathrm{H}_{13} \mathrm{O}_{4}\right]$ with 22 atoms and the optimized structure is shown in Fig. 1b. All DFT calculations were performed using the standard implementation of the ONIOM method in Gaussian 09 program package. Full geometry optimizations were carried out with the ONIOM (M06L/6-31+G**: MNDO) method for all clusters by following the DFT studies of Zhao \& Truhlar et al. [6] and Panjan et al. [7]. They found M06L method to be accurate for zeolite based systems.

In the present work, the effects of three dimensional network structures on bond length and bond angle were analyzed using a 3D:ZSM-5 and a BASCC.

\section{RESULTS AND DISCUSSION}

The specific bond length and bond angles selected in the 3D:ZSM-5 and the BASCC are shown in Tables 1 and 2, respectively. The most crucial bond present in the active site of the optimized structure of 3D:ZSM-5 (Fig. 1a) was A111-O12 and the corresponding bond in the optimized structure of BASCC (Fig. 1b) was A12-O3. The bond length of Al11-O12 was 1.70 $\AA$ in 3D:ZSM-5 and it became enhanced to $1.93 \AA$ in BASCC. This significant increase in bond length was exclusively due to the absence of three dimensional network structures. Another significant similar change in bond length was also identified in O10-Si19 (3D:ZSM-5) and the corresponding O3-Si5 (BASCC). The bond length of O10-Si19 was $1.62 \AA$ and that of O3-Si5 was $1.73 \AA$. These bond length related revelations were further supported from the results of bond angle analysis. There was a large increase in the $\mathrm{AlOH}$ bond angle in BASCC. In case of the 3D:ZSM-5 cluster, the Al11O12 H13 bond angle was identified to be $106.9^{\circ}$ whereas the corresponding bond angle in the BASCC was identified to be $122.8^{\circ}$. This signifi- 


\begin{tabular}{|c|c|c|c|c|c|c|c|}
\hline \multicolumn{8}{|c|}{$\begin{array}{l}\text { TABLE-1 } \\
\text { BOND LENGTHS AND BOND ANGLES OBTAINED FOR 3D:ZSM-5 CLUSTER FOR } \\
\text { ANALYZING THE EFFECT OF THREE DIMENSIONAL NETWORK STRUCTURE ON ZSM-5 }\end{array}$} \\
\hline \multicolumn{5}{|c|}{ Bond length $(\AA)$} & \multicolumn{3}{|c|}{ Bond angle $\left({ }^{\circ}\right)$} \\
\hline $\mathrm{Al}_{11}-\mathrm{O}_{10}$ & $\mathrm{O}_{12}-\mathrm{Si}_{14}$ & $\mathrm{O}_{10}-\mathrm{Si}_{19}$ & $\mathrm{Al}_{11}-\mathrm{O}_{10}$ & $\mathrm{O}_{12}-\mathrm{H}_{13}$ & $\mathrm{Al}_{11} \mathrm{O}_{12} \mathrm{H}_{13}$ & $\mathrm{Si}_{14} \mathrm{O}_{12} \mathrm{H}_{13}$ & $\mathrm{Al}_{11} \mathrm{O}_{12} \mathrm{Si}_{14}$ \\
\hline 1.7095 & 1.6964 & 1.6264 & 1.7095 & 0.9679 & 106.948 & 117.205 & 123.487 \\
\hline
\end{tabular}

TABLE-2

BOND LENGTH AND BOND ANGLES IN BASCC

\begin{tabular}{ccccc|ccc}
\hline \multicolumn{3}{c}{ Bond length $(\AA)$} & \multicolumn{3}{c}{ Bond angle $\left(^{\circ}\right)$} \\
\hline $\mathrm{Al}_{2}-\mathrm{O}_{1}$ & $\mathrm{O}_{1}-\mathrm{Si}_{10}$ & $\mathrm{O}_{3}-\mathrm{Si}_{5}$ & $\mathrm{Al}_{2}-\mathrm{O}_{3}$ & $\mathrm{O}_{3}-\mathrm{H}_{4}$ & $\mathrm{Al}_{2} \mathrm{O}_{3} \mathrm{H}_{4}$ & $\mathrm{Si}_{5} \mathrm{O}_{3} \mathrm{H}_{4}$ & $\mathrm{Al}_{2} \mathrm{O}_{3} \mathrm{Si}_{5}$ \\
\hline 1.7002 & 1.6247 & 1.7349 & 1.9325 & 0.9618 & 122.885 & 117.298 & 119.194 \\
\hline
\end{tabular}
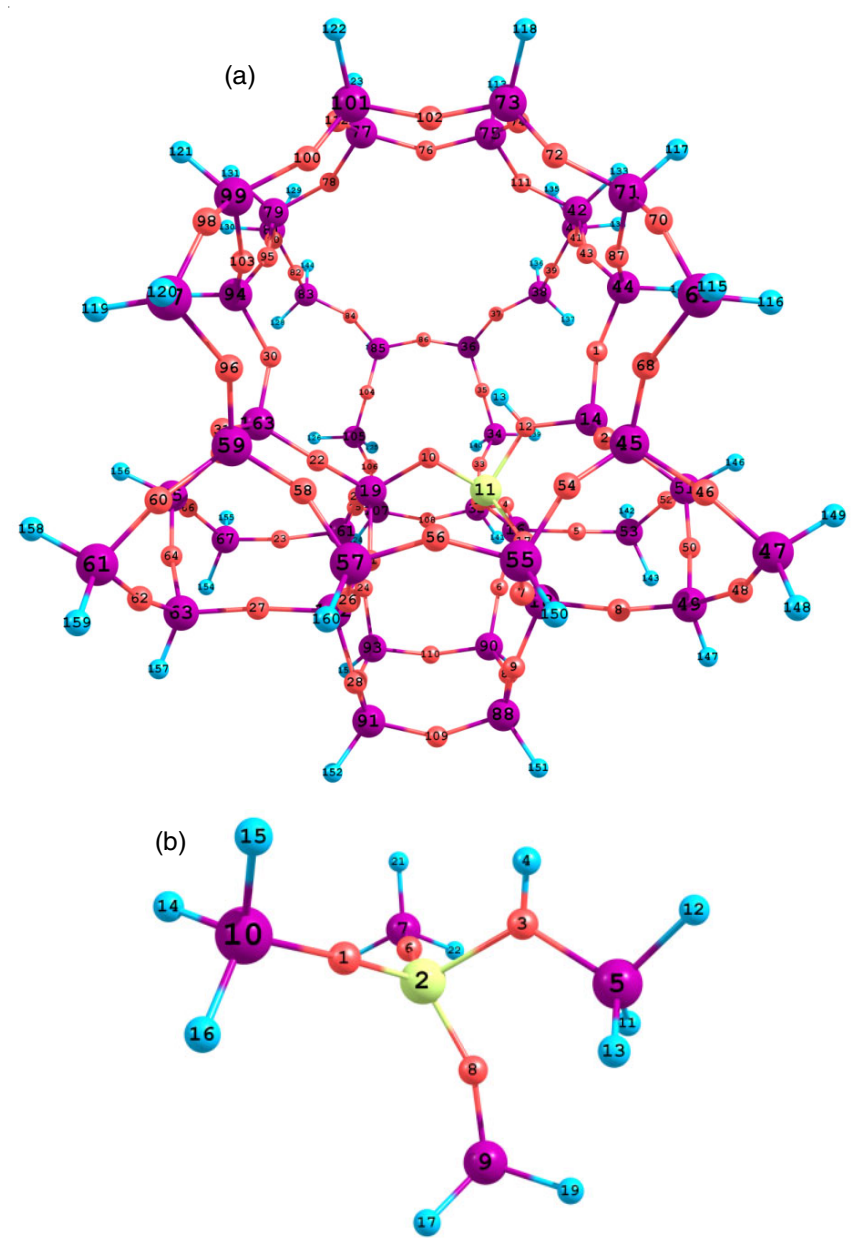

Fig. 1. Optimized structure of (a) 3D:ZSM-5 and (b) BASCC

cant increase in the bond angle and bond length in BASCC was again established to be exclusively due to the absence of the effect of three dimensional network structure(s). Thus the present study also indicates the necessity of analyzing the three dimensional structures for these purposes than the conventional simple cluster analysis as extensively reported. The optimized geometry of the ZSM-5 and CO adsorbed ZSM-5 are included in Figs. 1(a) and 2, respectively. The identification HOMO and LUMO orbitals generally give good idea about the reactivity and active site of the compounds [8]. The HOMO and LUMO were identified in order to understand the active site present in 3D:ZSM-5. Initially the BASCC was selected and the HOMO and LUMO orbitals were identified. In BASCC, the HOMO was identified at -0.2513 au and the LUMO at -0.0504 au as

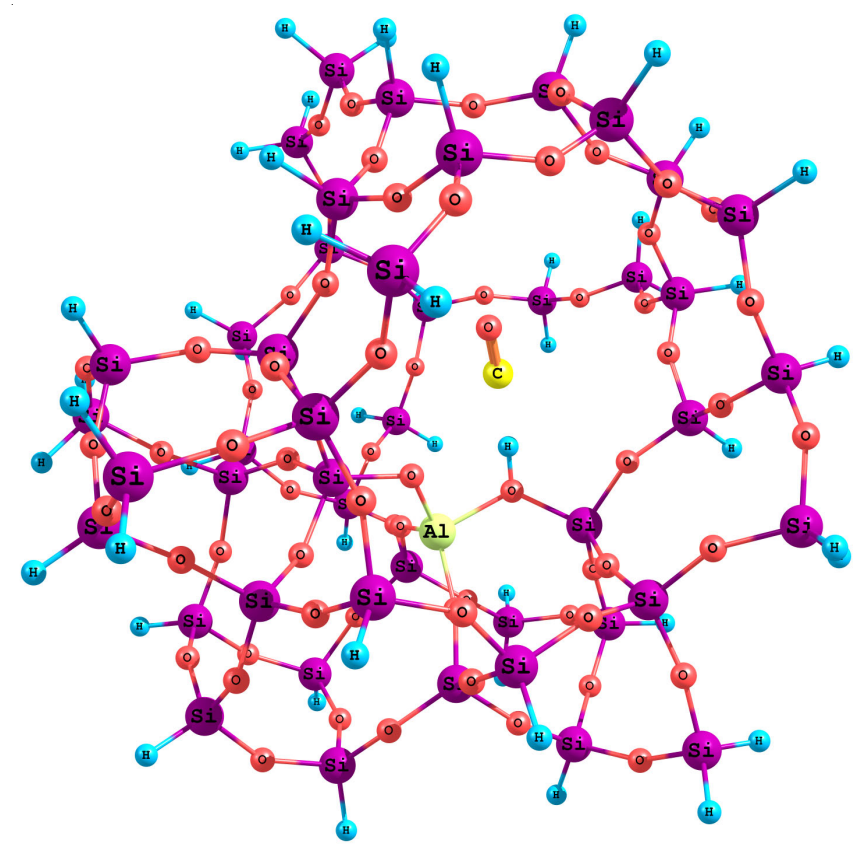

Fig. 2. Optimized geometry of ZSM-5 after CO adsorption

shown in Fig. 5(a) \& (b), respectively. The LUMO was found preferentially located at the Brønsted acid site. The active site present in the 3D:ZSM-5 was established by analyzing the HOMO and LUMO orbitals. The HOMO of the 3D:ZSM-5 was identified at -0.2596 au and the corresponding LUMO was identified at -0.0425 au. Interestingly, the LUMO was found preferentially located at the Brønsted acid site in the 3D:ZSM-5 with 163 atoms. These results again revealed that the active centre is concentrated at the Brønsted acid site in 3D: ZSM-5. The LUMO and HOMO orbitals present in 3D:ZSM-5 cluster are shown in Figs. 3 and 6, respectively. On completion of the establishment of the effect of the three dimensional structures and the nature of the catalytically active centres, the studies on the effect of adsorption of $\mathrm{CO}$ became apparent. Accordingly the shifting or relocation of the LUMO orbital from the Brønsted acid site to $\mathrm{CO}$ was required to be established. A study was carried out and the results are summarized in Figs. 3 and 4. The LUMO orbital was present at the Brønsted acid site in 3D:ZSM-5, but it got relocated due to adsorption of $\mathrm{CO}$. Finally it got relocated on adsorbed CO. Accordingly the HOMO and LUMO orbitals were identified at -0.2589 au and $-0.0838 \mathrm{au}$, respectively. The HOMO and LUMO orbitals of the adsorbed CO are included in Figs. 6(B) and 4, respectively. 


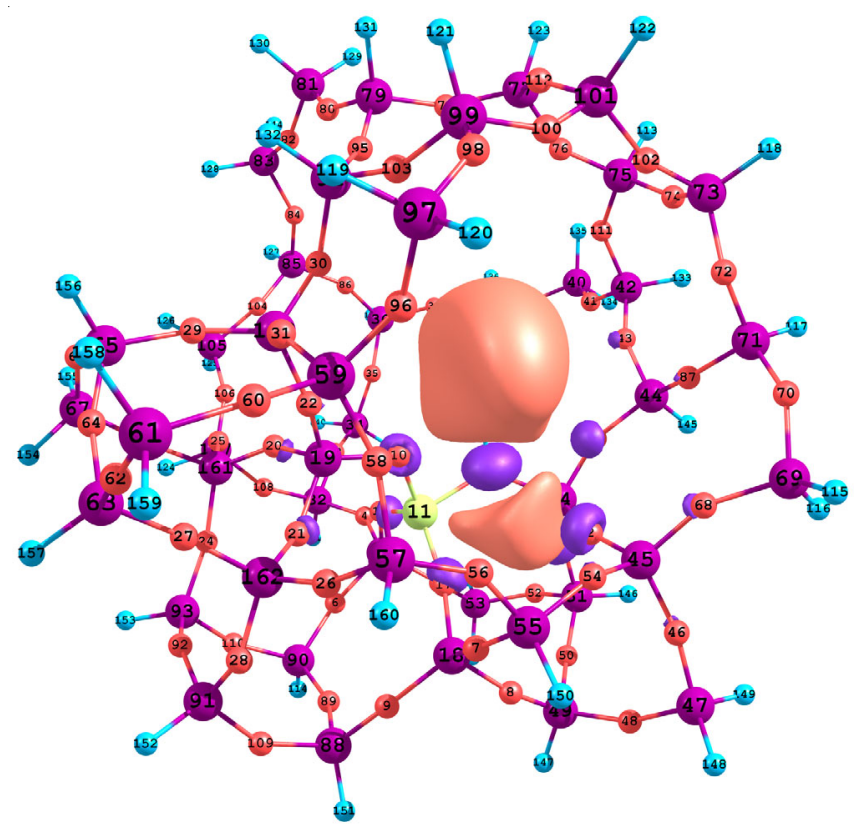

Fig. 3. LUMO orbital of ZSM-5 at the Brønsted acid site

Aihara [9] reported that the chemical reactivity could be directly correlated with HOMO-LUMO band gap. Accordingly we systematically analyzed the HOMO and LUMO orbitals in 3D:ZSM-5. The HOMO-LUMO gap in the free CO molecule was $7.58 \mathrm{eV}$ but it was $4.76 \mathrm{eV}$ in CO adsorbed ZSM-5. The significant reduction in the HOMO-LUMO energy gap in the $\mathrm{CO}$ adsorbed ZSM-5 revealed that the adsorbed CO molecule was more reactive compared to free $\mathrm{CO}$. Thus the active site stated to be concentrated on the Brønsted acid site in 3D: ZSM5 was again supported from the HOMO-LUMO energy gap analysis.

\section{Conclusion}

The proof of superior reactivity of the Brønsted acid site in 3D:ZSM-5 zeolite due to the preferential occurrence of

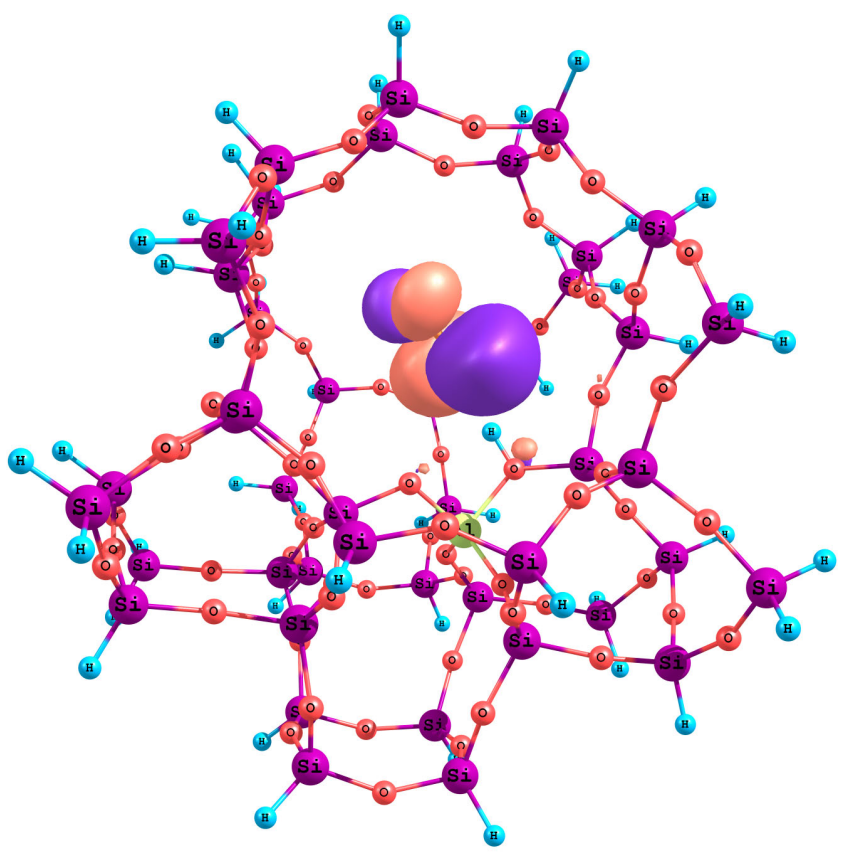

Fig. 4. Shifting of HOMO orbital from Brønsted acid site to adsorbed CO

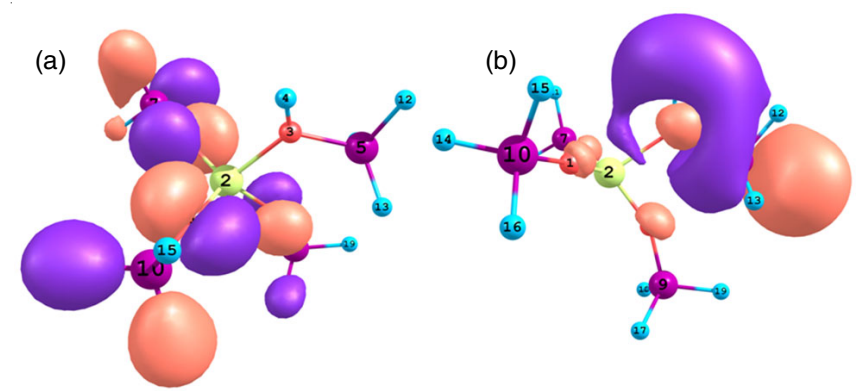

Fig. 5. HOMO of BASCC (a) and LUMO orbital of BASCC (b)

LUMO orbital at the Brønsted acid site was first established and the relocation of LUMO orbital from the Brønsted acid
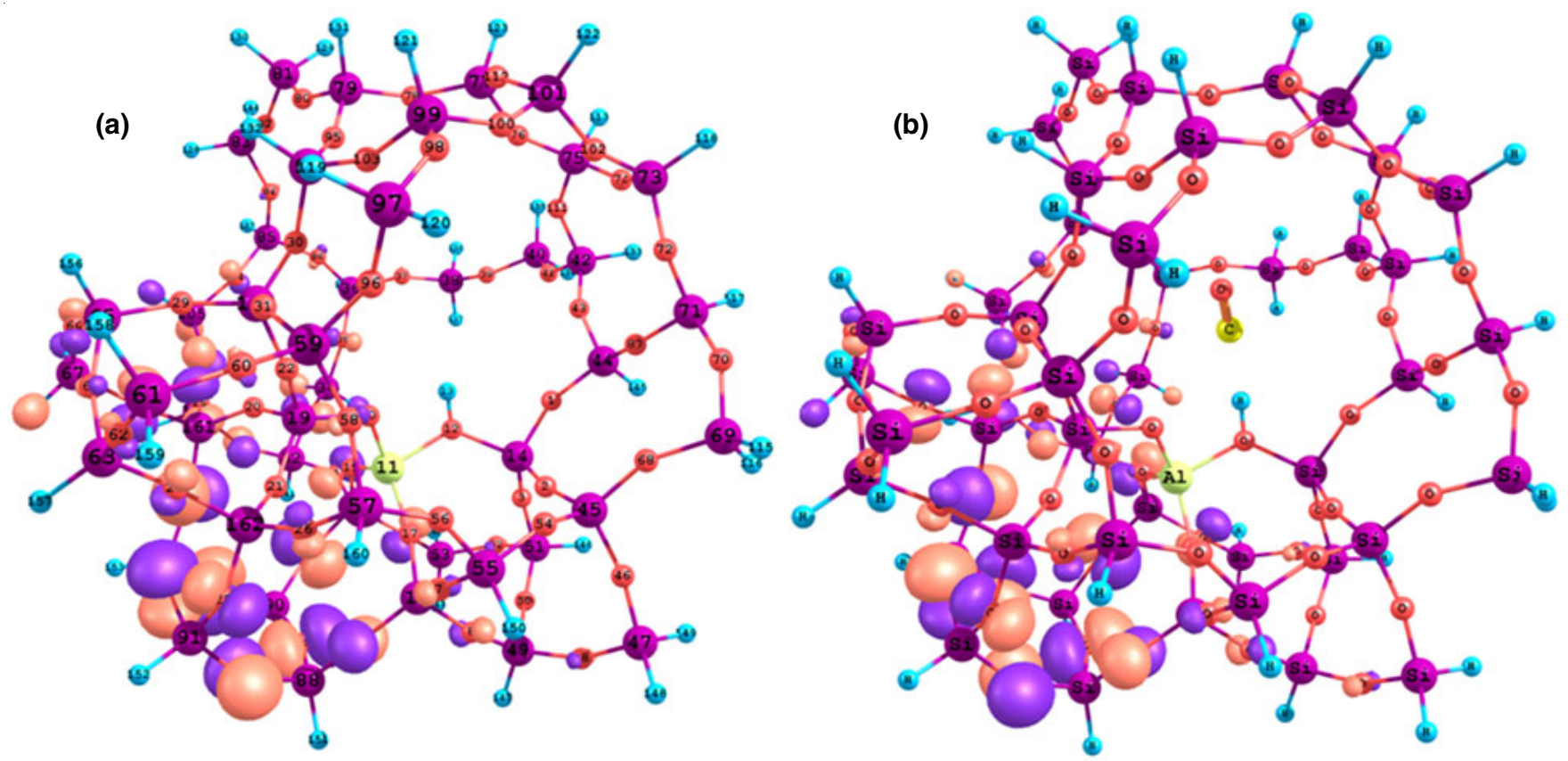

Fig. 6. HOMO orbital of (a) 3D:ZSM-5 and (b) CO adsorbed 3D:ZSM-5 
site to adsorbed carbon monoxide was accordingly verified. The significant lowering of band gap of carbon monoxide (CO) adsorbed on 3D:ZSM-5 compared to free $\mathrm{CO}$ revealed that adsorbed CO (haemo-toxic, stable) would be more reactive. Moreover, the effect of three dimensional structures on the activity of ZSM-5 has also been established based on bond length and bond angle computations. These revelations focus on the requirement of tuning of ZSM-5, one of the most important industrial catalysts, with concentrated Brønsted acid sites along with extensive network structures as far as possible.

\section{REFERENCES}

1. W. Cha, N.C. Jeong, S. Song, H. Park, T.C. Thanh Pham, R. Harder, B. Lim, G. Xiong, D. Ahn, I. McNulty, J. Kim, K.B. Yoon, I.K. Robinson and H. Kim, Nat. Mater., 12, 729 (2013);

https://doi.org/10.1038/nmat3698.
2. C. Li, H. Wang, S.S. Zhu, G.B. Liu and J.H. Wu, J. Fuel Chem. Technol., 45, 1088 (2017); https://doi.org/10.1016/S1872-5813(17)30051-8.

3. I.L.C. Buurmans, J. Ruiz-Martínez, W.V. Knowles, D. van der Beek, J.A. Bergwerff, E.T.C. Vogt and B.M. Weckhuysen, Nat. Chem., 3, 862 (2011); https://doi.org/10.1038/nchem.1148.

4. A. Corma, Chem. Rev., 95, 559 (1995); https://doi.org/10.1021/cr00035a006.

5. J. Van der Mynsbrugge, K. Hemelsoet, M. Vandichel, M. Waroquier and V. Van Speybroeck, J. Phys. Chem. C, 116, 5499 (2012); https://doi.org/10.1021/jp2123828.

6. Y. Zhao and D.G. Truhlar, J. Phys. Chem. C, 112, 6860 (2008); https://doi.org/10.1021/jp7112363.

7. W. Panjan, J. Sirijaraensre, C. Warakulwit, P. Pantu and J. Limtrakul, Phys. Chem. Chem. Phys., 14, 16588 (2012); https://doi.org/10.1039/c2cp42066j.

8. S. Sakkiah and K.W. Lee, Acta Pharmacol. Sin., 33, 964 (2012); https://doi.org/10.1038/aps.2012.21.

9. J.I. Aihara, Phys. Chem. Chem. Phys., 2, 3121 (2000); https://doi.org/10.1039/b002601h. 